\title{
Numerical solution of Rosenau-KdV equation using subdomain finite element method
}

\author{
S. Battal Gazi Karakoc ${ }^{1}$ and Turgut $A k^{2}$ \\ ${ }^{1}$ Department of Mathematics, Nevsehir Haci Bektas Veli University, Nevsehir, Turkey \\ ${ }^{2}$ Department of Transportation Engineering, Yalova University, Yalova, Turkey
}

Received: 1 December 2015, Revised: 24 December 2015, Accepted: 30 December 2015

Published online: 11 February 2016.

\begin{abstract}
In this paper, a numerical solution of the Rosenau-Korteweg-de Vries (Rosenau-KdV) equation, based on subdomain method using sextic B-spline is utilized to simulate the motion of single solitary wave. The two invariants of the motion are worked out to define the conservation properties. $L_{2}$ and $L_{\infty}$ error norms are used to measure differences between the analytical and numerical solutions. Applying the von-Neumann stability analysis, the proposed method is illustrated to be unconditionally stable. The method is applied on three test examples, and the computed numerical solutions are in good agreement with the result available in literature as well as with exact solutions. The numerical results depict that the scheme is efficient and feasible.
\end{abstract}

Keywords: Rosenau-KdV equation, solitons, shallow water, subdomain.

\section{Introduction}

Special forms of water waves known as dispersive shallow water waves are receiving quite a bit of attention during the past few years $[1,2,3,4,5,6,7,8,9,10]$. Some of these models are Rosenau-KdV equation, Rosenau-Kawahara equation and Rosenau-KdV-RLW equation. In these few models, it is the dispersion that dominates but nevertheless maintains a delicate balance with nonlinearity which ensures the propagation of dispersive solitary waves. Thus far, there are many analytical results that are reported in this context. This paper will change its focus and gear towards numerical simulations of the model so that the model can be analyzed from a different perspective.

The Korteweg-de Vries equation is the milestone of the nonlinear science derived by Korteweg and de Vries as the following $[11,2,13,14,15,16]$ :

$$
U_{t}+U U_{x}+U_{x x x}=0
$$

Eq. (1) is a fundamental mathematical model to describe of weakly nonlinear long wave propagation in dispersive media. The equation is an significant partial differential equation which arises in the study of many physical phenomena. In the study of the dynamics of dense discrete systems, the case of wave-wave and wave-wall interactions can not be described using the well-known KdV equation. Philip Rosenau developed a formulation to treat the dynamics of dense discrete systems to get over this deficiency of the $\mathrm{KdV}$ equation $[17,18]$. Rosenau equation is stated by

$$
U_{t}+U_{x x x x t}+U_{x}+U U_{x}=0
$$


On the numerical solutions of the Eq. (2), many studies have been performed by the scholars [19, 20,21, 22, 23]. Then, for the further consideration of the nonlinear wave, Jin-Ming Zuo developed the following so-called Rosenau-KdV equation and discussed the solitary wave solutions and periodic solutions of its with the sine-cosine and the tanh methods in Ref.[24]

$$
U_{t}+U_{x x x x t}+U_{x}+U U_{x}+U_{x x x}=0
$$

where $U_{x x x}$ is the viscous term and subscripts $x$ and $t$ denote differentiation. Recently, the solitary solutions were examined for the generalized Rosenau-KdV eqaution [25,26,27]. Two invariants were given for the equation in [25,26]. Especially, in Ref. [26] both the singular single soliton solution is derived by the ansatz method and the perturbation theory is applied to obtain the adiabatic parameter dynamics of the water waves. The ansatz method is carried out to obtain the topological soliton (shock) solution of the generalized Rosenau-KdV equation [27]. The $G^{\prime} / G$ expansion, ansatz and the exp-function methods are implemented to achieve several solutions to the equation [28]. A conservative three-level linear finite difference scheme for the numerical solution of the initial-bounday value problem of the Rosenau-KdV equatin is suggested [29]. A mathematical model to obtain the solution of the nonlinear wave by coupling the Rosenau-KdV equation and the Rosenau-RLW equation is proposed. A numerical tool is applied to the model by using a three-level average implicit finite difference technique. The fundamental conservative properties of the equation are preserved by the presented numerical scheme, and the existence and uniqueness of the numerical solution are proved [30].

In the present paper, a numerical scheme based on the sextic B-spline subdomain method has been set up for solving the Rosenau-KdV equation with a variant of both initial and boundary conditions. This work is set out as the following. In Section 2, sextic B-spline subdomain scheme is presented. In Section 3, stability analysis of the scheme is considered. In Section 4, numerical examples and results of the equation are obtained. Finally in Section 5, a summary is given at the end of the paper.

\section{Sextic B-spline subdomain finite element method}

Consider the Rosenau-KdV Eq. (3) is given by the following initial and boundary conditions:

$$
\begin{aligned}
U(x, 0)=f(x) \quad a \leq x \leq b, \\
U(a, t)=0, \quad U(b, t)=0, \\
U_{x}(a, t)=0, \quad U_{x}(b, t)=0, \\
U_{x x}(a, t)=0, \quad U_{x x}(b, t)=0, \quad t>0 .
\end{aligned}
$$

The interval $[a, b]$ is divided by uniformly sized finite elements of equal length $h$ by the knots $x_{m}$ like that $a=x_{0}<x_{1} \cdots<x_{N}=b$. Let $\phi_{m}(x)$ be sextic B-splines with knots at the points $x_{m}, m=0,1, \ldots, N$. The set of splines $\left\{\phi_{-3}, \phi_{-2}, \ldots, \phi_{N+1}, \phi_{N+2}\right\}$ forms a basis for functions determined over $[a, b]$. So a global approximation $U_{N}(x, t)$ to the exact solution $U(x, t)$ can be stated in accordance with the sextic B-splines as:

$$
U_{N}(x, t)=\sum_{m=-3}^{N+2} \delta_{m}(t) \phi_{m}(x),
$$


where $\delta_{m}$ are time dependent quantities to be determined from both boundary and weighted residual conditions. Each sextic B-spline covers seven elements so that each element $\left[x_{m}, x_{m+1}\right]$ is covered by seven splines. Sextic B-splines $\phi_{m}(x)$, $(m=-3(1) N+2)$, at the knots $x_{m}$ which form a basis over the interval $[a, b]$ are determined by the relationships Ref.[31];

$$
\phi_{m}(x)=\frac{1}{h^{6}}\left\{\begin{array}{lr}
\left(x-x_{m-3}\right)^{6}, & x \in\left[x_{m-3}, x_{m-2}\right], \\
\left(x-x_{m-3}\right)^{6}-7\left(x-x_{m-2}\right)^{6}, & x \in\left[x_{m-2}, x_{m-1}\right], \\
\left(x-x_{m-3}\right)^{6}-7\left(x-x_{m-2}\right)^{6}+21\left(x-x_{m-1}\right)^{6}, & x \in\left[x_{m-1}, x_{m}\right], \\
\left(x-x_{m-3}\right)^{6}-7\left(x-x_{m-2}\right)^{6}+21\left(x-x_{m-1}\right)^{6}-35\left(x-x_{m}\right)^{6}, & x \in\left[x_{m}, x_{m+1}\right], \\
\left(x-x_{m+4}\right)^{6}-7\left(x-x_{m+3}\right)^{6}+21\left(x-x_{m+2}\right)^{6}, & x \in\left[x_{m+1}, x_{m+2}\right], \\
\left(x-x_{m+4}\right)^{6}-7\left(x-x_{m+3}\right)^{6}, & x \in\left[x_{m+2}, x_{m+3}\right], \\
\left(x-x_{m+4}\right)^{6}, & x \in\left[x_{m+3}, x_{m+4}\right], \\
0, & \text { otherwise, }
\end{array}\right.
$$

where $h=\left(x_{m+1}-x_{m}\right)$. Using (6) and (7), the nodal values $U$ and its first, second and third derivatives at the knots $x_{m}$ are obtained as the following:

$$
\begin{aligned}
& U_{m}=U\left(x_{m}\right)=\delta_{m-3}+57 \delta_{m-2}+302 \delta_{m-1}+302 \delta_{m}+57 \delta_{m+1}+\delta_{m+2}, \\
& U_{m}^{\prime}=U^{\prime}\left(x_{m}\right)=\frac{6}{h}\left(-\delta_{m-3}-25 \delta_{m-2}-40 \delta_{m-1}+40 \delta m+25 \delta_{m+1}+\delta_{m+2}\right), \\
& U_{m}^{\prime \prime}=U^{\prime \prime}\left(x_{m}\right)=\frac{30}{h^{2}}\left(\delta_{m-3}+9 \delta_{m-2}-10 \delta_{m-1}-10 \delta_{m}+9 \delta_{m+1}+\delta_{m+2}\right) \\
& U_{m}^{\prime \prime \prime}=U^{\prime \prime \prime}\left(x_{m}\right)=\frac{120}{h^{3}}\left(-\delta_{m-3}-\delta_{m-2}+8 \delta_{m-1}-8 \delta_{m}+\delta_{m+1}+\delta_{m+2}\right)
\end{aligned}
$$

A typical finite interval $\left[x_{m}, x_{m+1}\right]$ is turned into the interval $[0,1]$ by local coordinates $\xi$ regarding the global coordinates

$$
h \xi=x-x_{m}, \quad 0 \leq \xi \leq 1,
$$

so the sextic B-spline shape functions over the element $[0,1]$ can be defined as $\phi^{e}=\left(\phi_{m-3}, \phi_{m-2}, \phi_{m-1}, \phi_{m}, \phi_{m+1}, \phi_{m+2}, \phi_{m+3}\right)$

$$
\phi^{e}=\left\{\begin{array}{l}
\phi_{m-3}=1-6 \xi+15 \xi^{2}-20 \xi^{3}+15 \xi^{4}-6 \xi^{5}+\xi^{6}, \\
\phi_{m-2}=57-150 \xi+135 \xi^{2}-20 \xi^{3}-45 \xi^{4}+30 \xi^{5}-6 \xi^{6}, \\
\phi_{m-1}=302-240 \xi-150 \xi^{2}+160 \xi^{3}+30 \xi^{4}-60 \xi^{5}+15 \xi^{6} \\
\phi_{m}=302+240 \xi-150 \xi^{2}-160 \xi^{3}+30 \xi^{4}+60 \xi^{5}-20 \xi^{6}, \\
\phi_{m+1}=57+150 \xi+135 \xi^{2}+20 \xi^{3}-45 \xi^{4}-30 \xi^{5}+156 \xi^{6}, \\
\phi_{m+2}=1+6 \xi+15 \xi^{2}+20 \xi^{3}+15 \xi^{4}+6 \xi^{5}-6 \xi^{6}, \\
\phi_{m+3}=\xi^{6} .
\end{array}\right.
$$

Since all splines other than $\phi_{m-3}(x), \phi_{m-2}(x), \phi_{m-1}(x), \phi_{m}(x), \phi_{m+1}(x), \phi_{m+2}(x), \phi_{m+3}(x)$ are zero over the element $[0,1]$. Approximation (7) over this element can be noted down in terms of basis functions (10) as

$$
U_{N}(\xi, t)=\sum_{j=m-3}^{m+3} \delta_{j}(t) \phi_{j}(\xi)
$$

where $\delta_{m-3}, \delta_{m-2}, \delta_{m-1}, \delta_{m}, \delta_{m+1}, \delta_{m+2}, \delta_{m+3} \quad$ act $\quad$ as element parameters and B-splines $\phi_{m-3}(x), \phi_{m-2}(x), \phi_{m-1}(x), \phi_{m}(x), \phi_{m+1}(x), \phi_{m+2}(x)$ and $\phi_{m+3}(x)$ as element shape functions. Application of subdomain 
method to the Eq. (3) with weight function

$$
W_{m}(x)= \begin{cases}1, & x \in\left[x_{m}, x_{m+1}\right] \\ 0, & \text { otherwise }\end{cases}
$$

creates the weak form

$$
\int_{x_{m}}^{x_{m+1}} 1 .\left(U_{t}+U_{x x x x t}+U_{x}+U U_{x}+U_{x x x}\right) d x=0 .
$$

Substituting the transformation (10) into weak form (13) and integrating Eq. (13) term by term with some manipulation by parts, brings on

$$
\begin{aligned}
& \frac{h}{7}\left(\dot{\delta}_{m-3}+120 \dot{\delta}_{m-2}+1191 \dot{\delta}_{m-1}+2416 \dot{\delta}_{m}+1191 \dot{\delta}_{m+1}+120 \dot{\delta}_{m+2}+\dot{\delta}_{m+3}\right) \\
& +\frac{120}{h^{3}}\left(\dot{\delta}_{m-3}-9 \dot{\delta}_{m-1}+16 \dot{\delta}_{m}-9 \dot{\delta}_{m+1}+\dot{\delta}_{m+3}\right) \\
& +\left(-\delta_{m-3}-56 \delta_{m-2}-245 \delta_{m-1}+245 \delta_{m+1}+56 \delta_{m+2}+\delta_{m+3}\right) \\
& +Z_{m}\left(-\delta_{m-3}-56 \delta_{m-2}-245 \delta_{m-1}+245 \delta_{m+1}+56 \delta_{m+2}+\delta_{m+3}\right) \\
& +\frac{30}{h^{2}}\left(-\delta_{m-3}-8 \delta_{m-2}+19 \delta_{m-1}-19 \delta_{m+1}+8 \delta_{m+2}+\delta_{m+3}\right)=0
\end{aligned}
$$

where the dot indicates differentiation with respect to $t$ and

$$
Z_{m}=\delta_{m-3}+57 \delta_{m-2}+302 \delta_{m-1}+302 \delta_{m}+57 \delta_{m+1}+\delta_{m+2}
$$

If time parameters $\delta_{m}$ and its time derivatives $\dot{\delta}_{m}$ in Eq. (13) are discretized by the Crank-Nicolson and forward difference approach respectively,

$$
\delta=\frac{\delta_{m}^{n}+\delta_{m}^{n+1}}{2}, \quad \dot{\delta}_{m}=\frac{\delta_{m}^{n+1}-\delta_{m}^{n}}{\Delta t}
$$

we obtain a recurrence relationship between two time levels $n$ and $n+1$ relating two unknown parameters $\delta_{i}^{n+1}, \delta_{i}^{n}$, $i=m-3, m-2, \ldots, m+3$,

$$
\begin{aligned}
& \alpha_{m 1} \delta_{m-3}^{n+1}+\alpha_{m 2} \delta_{m-2}^{n+1}+\alpha_{m 3} \delta_{m-1}^{n+1}+\alpha_{m 4} \delta_{m}^{n+1}+\alpha_{m 5} \delta_{m+1}^{n+1}+\alpha_{m 6} \delta_{m+2}^{n+1}+\alpha_{m 7} \delta_{m+3}^{n+1} \\
& \alpha_{m 7} \delta_{m-3}^{n}+\alpha_{m 6} \delta_{m-2}^{n}+\alpha_{m 5} \delta_{m-1}^{n}+\alpha_{m 4} \delta_{m}^{n}+\alpha_{m 3} \delta_{m+1}^{n}+\alpha_{m 2} \delta_{m+2}^{n}+\alpha_{m 1} \delta_{m+3}^{n}
\end{aligned}
$$

where

$$
\begin{array}{lrl}
\alpha_{m 1}=1-E\left(1+Z_{m}\right)-M+K, & \alpha_{m 2}=120-56 E\left(1+Z_{m}\right)-8 M, \\
\alpha_{m 3}=1191-245 E\left(1+Z_{m}\right)+19 M-9 K, & \alpha_{m 4}=2416+16 K, \\
\alpha_{m 5}=1191+245 E\left(1+Z_{m}\right)-19 M-9 K, & \alpha_{m 6}=120+56 E\left(1+Z_{m}\right)+8 M, \\
\alpha_{m 7}=1+E\left(1+Z_{m}\right)+M+K, & m=0,1, \ldots, N-1,
\end{array}
$$

and

$$
E=\frac{7 \Delta t}{2 h}, \quad M=\frac{105 \Delta t}{h^{3}}, \quad K=\frac{840}{h^{4}}
$$

The system (16) consists of $N$ linear equation in $N+6$ unknowns $\left(\delta_{-3}, \delta_{-2}, \ldots, \delta_{N+1}, \delta_{N+2}\right)$. To get a solution of this system, we need six additional constraints. These are obtained from the boundary conditions (5). These conditions provide us elimination of the parameters $\delta_{-3}, \delta_{-2}, \delta_{-1}, \delta_{N}, \delta_{N+1}$ and $\delta_{N+2}$ from the system (16) which then becomes a matrix equation for the $N$ unknowns $d=\left(\delta_{0}, \delta_{1}, \ldots, \delta_{N-1}\right)$ of the form

$$
A d^{n+1}=B d^{n}
$$


A lumped value for $Z_{m}$ is obtained from $\left(U_{m}+U_{m+1}\right) / 2$ as

$$
Z_{m}=\frac{1}{2}\left(\delta_{m-3}^{n}+58 \delta_{m-2}^{n}+359 \delta_{m-1}+604 \delta_{m}^{n}+359 \delta_{m+1}^{n}+58 \delta_{m+2}^{n}+\delta_{m+3}^{n}\right) .
$$

The resulting system can be effectively solved with a variant of the Thomas algorithm, and we need an inner iteration $\delta^{n *}=\delta^{n}+\frac{1}{2}\left(\delta^{n}-\delta^{n-1}\right)$ at each time step to cope with the non-linear term $Z_{m}$. A typical member of the matrix system (16) can be written in terms of the nodal parameters $\delta_{m}^{n}$ as

$$
\begin{aligned}
& \gamma_{1} \delta_{m-3}^{n+1}+\gamma_{2} \delta_{m-2}^{n+1}+\gamma_{3} \delta_{m-1}^{n+1}+\gamma_{4}+\gamma_{5} \delta_{m+1}^{n+1}+\gamma_{6} \delta_{m+2}^{n+1}+\gamma_{7} \delta_{m+3}^{n+1}= \\
& \gamma_{7} \delta_{m-3}^{n}+\gamma_{6} \delta_{m-2}^{n}+\gamma_{5} \delta_{m-1}^{n}+\gamma_{4}+\gamma_{3} \delta_{m+1}^{n}+\gamma_{2} \delta_{m+2}^{n}+\gamma_{1} \delta_{m+3}^{n}
\end{aligned}
$$

where

$$
\begin{array}{ll}
\gamma_{1}=\alpha-\beta-\lambda+\mu, & \gamma_{2}=120 \alpha-56 \beta-8 \lambda, \\
\gamma_{3}=1191 \alpha-245 \beta+19 \lambda-9 \mu, & \gamma_{4}=2416 \alpha+16 \mu, \\
\gamma_{5}=1191 \alpha+245 \beta-19 \lambda-9 \mu, & \gamma_{6}=120 \alpha+56 \beta+8 \lambda, \\
\gamma_{7}=\alpha+\beta+\lambda+\mu, &
\end{array}
$$

and

$$
\alpha=1, \beta=E\left(1+Z_{m}\right), \lambda=M, \mu=K \quad m=0,1, \ldots, N-1 .
$$

To start the iteration relation system equation (15), initial parameters must be determined by the aid of initial condition and six boundary conditions as the following:

$$
\begin{aligned}
U_{N}\left(x_{m}, 0\right) & =\delta_{m-3}^{0}+57 \delta_{m-2}^{0}+302 \delta_{m-1}^{0}+302 \delta_{m}^{0}+57 \delta_{m+1}^{0}+\delta_{m+2}^{0}=U\left(x_{m}, 0\right), \\
U_{N}^{\prime}(a, 0) & =-\delta_{-3}^{0}-25 \delta_{-2}^{0}-40 \delta_{-1}^{0}+40 \delta_{0}^{0}+25 \delta_{1}^{0}+\delta_{2}^{0}=0, \\
U_{N}^{\prime \prime}(a, 0) & =\delta_{-3}^{0}+9 \delta_{-2}^{0}-10 \delta_{-1}^{0}-10 \delta_{0}^{0}+9 \delta_{1}^{0}+\delta_{2}^{0}=0, \\
U_{N}^{\prime \prime \prime}(a, 0) & =-\delta_{-3}^{0}-\delta_{-2}^{0}+8 \delta_{-1}^{0}-8 \delta_{0}^{0}+\delta_{1}^{0}+\delta_{2}^{0}=0, \\
U_{N}^{\prime}(b, 0) & =-\delta_{N-3}^{0}-25 \delta_{N-2}^{0}-40 \delta_{N-1}^{0}+40 \delta_{N}^{0}+25 \delta_{N+1}^{0}+\delta_{N+2}^{0}=0, \\
U_{N}^{\prime \prime}(b, 0) & =\delta_{N-3}^{0}+9 \delta_{N-2}^{0}-10 \delta_{N-1}^{0}-10 \delta_{N}^{0}+9 \delta_{N+1}^{0}+\delta_{N+2}^{0}=0, \\
U_{N}^{\prime \prime \prime}(b, 0) & =-\delta_{N-3}^{0}-\delta_{N-2}^{0}+8 \delta_{N-1}^{0}-8 \delta_{N}^{0}+\delta_{N+1}^{0}+\delta_{N+2}^{0}=0 .
\end{aligned}
$$

Eliminating $\delta_{-3}^{0}, \delta_{-2}^{0}, \delta_{-1}^{0}, \delta_{N}^{0}, \delta_{N+1}^{0}, \delta_{N+2}^{0}$ from the system (16) we get $N \times N$ matrix system of the form

$$
W \delta^{0}=B
$$

where $W$ is




$\delta^{0}=\left[\delta_{0}^{0}, \delta_{1}^{0}, \ldots, \delta_{N-1}^{0}\right]^{T}$ and $B=\left[U\left(x_{0}, 0\right), U\left(x_{1}, 0\right), \ldots, U\left(x_{N-1}, 0\right)\right]^{T}$. This matrix system can be solved effectively by using a variant of Thomas algorithm.

\section{Stability analysis}

The stability analysis is based on the von-Neumann theory in which the growth factor of a typical Fourier mode

$$
\delta_{j}^{n}=\xi^{n} e^{i j k h}
$$

where $k$ is mode number and $h$ is the element size, is determined for a linearization of the numerical scheme. To carry out the stability analysis, the Rosenau-KdV equation needs to be linearized by supposing that the quantity $U$ in the non-linear term $U U_{x}$ is locally constant. Substituting the equation (23) into the scheme (20) we have

$$
\xi=\frac{a-i b}{a+i b}
$$

where

$$
\begin{aligned}
& a=(1208+8 \mu)+(1191-9 \mu) \cos (k h)+120 \cos (2 k h)+(1+\mu) \cos (3 k h), \\
& b=(245 \beta-19 \lambda) \sin (k h)+(56 \beta+8 \lambda) \sin (2 k h)+(\beta+\lambda) \sin (3 k h) .
\end{aligned}
$$

Taking the modulus of equation (24) gives $|\xi|=1$, hence we find that the scheme (20) is unconditionally stable.

\section{Numerical examples and results}

In this section, we consider the motion of single solitary wave and three test examples. Accuracy and efficiency of the method is measured by the error norm $L_{2}$

$$
L_{2}=\left\|U^{\text {exact }}-U_{N}\right\|_{2} \simeq \sqrt{h \sum_{J=1}^{N}\left|U_{j}^{\text {exact }}-\left(U_{N}\right)_{j}\right|^{2}},
$$

and the error norm $L_{\infty}$

$$
L_{\infty}=\left\|U^{\text {exact }}-U_{N}\right\|_{\infty} \simeq \max _{j}\left|U_{j}^{\text {exact }}-\left(U_{N}\right)_{j}\right|, j=1,2, \ldots, N-1 .
$$

Rosenau-KdV equation satisfies only two conservation laws given by Ref.[25]

$$
\begin{aligned}
& I_{1}=\int_{a}^{b} U d x \simeq h \sum_{J=1}^{N} U_{j}^{n} \\
& I_{2}=\int_{a}^{b}\left[U^{2}+\left(U_{x x}\right)^{2}\right] d x \simeq h \sum_{J=1}^{N}\left[\left(U_{j}^{n}\right)^{2}+\left(U_{x x}\right)_{j}^{n}\right]
\end{aligned}
$$

In the simulation of solitary wave motion, the invariants $I_{1}$ and $I_{2}$ are monitored to check the conversation of the numerical algorithm.

\subsection{The motion of single solitary wave}

For this problem, we consider solitary wave solution of Eq. (3) with the boundary conditions $U \rightarrow 0$ as $x \rightarrow \pm \infty$ and the initial condition

$$
U(x, 0)=\left(-\frac{35}{24}+\frac{35}{312} \sqrt{313}\right) \operatorname{sech}^{4}\left(\frac{1}{24} \sqrt{-26+2 \sqrt{313}} x\right) .
$$


Table 1: Comparison of the results for single solitary wave with amplitude $=0.52632, h=0.1, \Delta t=0.1,-70 \leq x \leq 100$.

\begin{tabular}{|c|c|c|c|c|c|c|}
\hline \multicolumn{2}{|c|}{$t$} & 0 & 10 & 20 & 30 & 40 \\
\hline \multirow[t]{2}{*}{$I_{1}$} & Present & 5.4981750556 & 5.4981749939 & 5.4981749598 & 5.4981749423 & 5.4981749335 \\
\hline & [29] & 5.4977225480 & 5.4977249365 & 5.4977287449 & 5.4977319638 & 5.4977342352 \\
\hline \multirow[t]{2}{*}{$I_{2}$} & Present & 1.9897841614 & 1.9897841614 & 1.9897841614 & 1.9897841614 & 1.9897841614 \\
\hline & [29] & 1.9845533653 & 1.9845950759 & 1.9846459641 & 1.9846798272 & 1.9847015013 \\
\hline \multirow[t]{4}{*}{$L_{2} \times 10^{3}$} & Present & 0.000000 & 0.356724 & 0.646705 & 0.902514 & 1.162489 \\
\hline & [29] & 0.000000 & 1.641934 & 3.045414 & 4.241827 & 5.297873 \\
\hline & [30] & - & - & 0.657830 & - & - \\
\hline & [30] & - & - & 0.443965 & - & - \\
\hline \multirow[t]{4}{*}{$L_{\infty} \times 10^{3}$} & Present & 0.000000 & 0.141639 & 0.244374 & 0.326169 & 0.411492 \\
\hline & [29] & 0.000000 & 0.631419 & 1.131442 & 1.533771 & 1.878952 \\
\hline & [30] & - & - & 0.254116 & - & - \\
\hline & [30] & - & - & 0.123727 & - & - \\
\hline
\end{tabular}

Note that the analytical solution of this problem can be written as

$$
U(x, t)=\left(-\frac{35}{24}+\frac{35}{312} \sqrt{313}\right) \operatorname{sech}^{4}\left[\frac{1}{24} \sqrt{-26+2 \sqrt{313}}\left(x-\left(\frac{1}{2}+\frac{1}{26} \sqrt{313}\right) t\right) .\right.
$$

For the numerical simulation of this problem, three sets of parameters have been chosen and discussed. Firstly, we have used the parameters space $\operatorname{step} h=0.1$ and time step $\Delta t=0.1$ over the problem domain $[-70,100]$ to coincide with those of earlier results $[29,30]$. So, the solitary wave has an amplitude 0.52632 and the run of the algorithm is carried up to time $t=40$ to obtain the invariants and error norms $L_{2}$ and $L_{\infty}$ at various times. Error norms $L_{2}, L_{\infty}$ and two invariants of the Rosenau-KdV equation are listed in Table 1. It is seen from the table that the error norms are found to be small enough. The percentage of the relative error of the conserved quantities $I_{1}$ and $I_{2}$ are calculated with respect to the conserved quantities at $t=0$. Percentage of relative changes of $I_{1}$ and $I_{2}$ are found to be $2.2211 \times 10^{-6} \%, 6 \times 10^{-10} \%$, respectively. Thus, the invariants remain almost constant during the computer run. Also, Table 1 exhibits a comparison of the values of the invariants and error norms obtained by the present method with those obtained by earlier results [29,30]. It can be seen from the Table 1 that the error norms obtained by the present method are smaller than given in Ref.[29] and almost the same in Ref.[30] at $t=20$. Figure 1 shows the motion of solitary wave with $h=0.1$ and $\Delta t=0.1$ at different time levels. It is observed that the soliton moves to the right at a constant speed and preserves its amplitude and shape with an increasing of time, as expected. The distributions of the errors at time $t=40$ are illustrated for solitary waves amplitudes 0.52632 in Figure 2.

For the second set, the parameters $h=0.05$ and $\Delta t=0.05$ with range $[-70,100]$ are taken to compare the results obtained by the present method with earlier results $[29,30]$. So, the solitary wave has amplitude 0.52632 and the simulations are run up to time $t=40$ to obtain the invariants and the error norms $L_{2}$ and $L_{\infty}$ at various times. Error norms $L_{2}$ and $L_{\infty}$ and conserved quantities are reported in Table 2 together with earlier results [29,30]. It can be easily seen from the table that the error norms obtained by the present method are smaller than given in Ref.[29] and almost the same in Ref.[30] at $t=20$. The agreement between numerical and analytic solution is excellent. Percentage of relative changes of $I_{1}$ and $I_{2}$ are found to be $9.171 \times 10^{-7} \%, 1.4 \times 10^{-9} \%$, respectively. The profiles of the solitary wave at some discrete times are shown in Figure 3. The distributions of the errors at time $t=40$ are drawn for solitary waves amplitudes 0.52632 in Figure 4. 


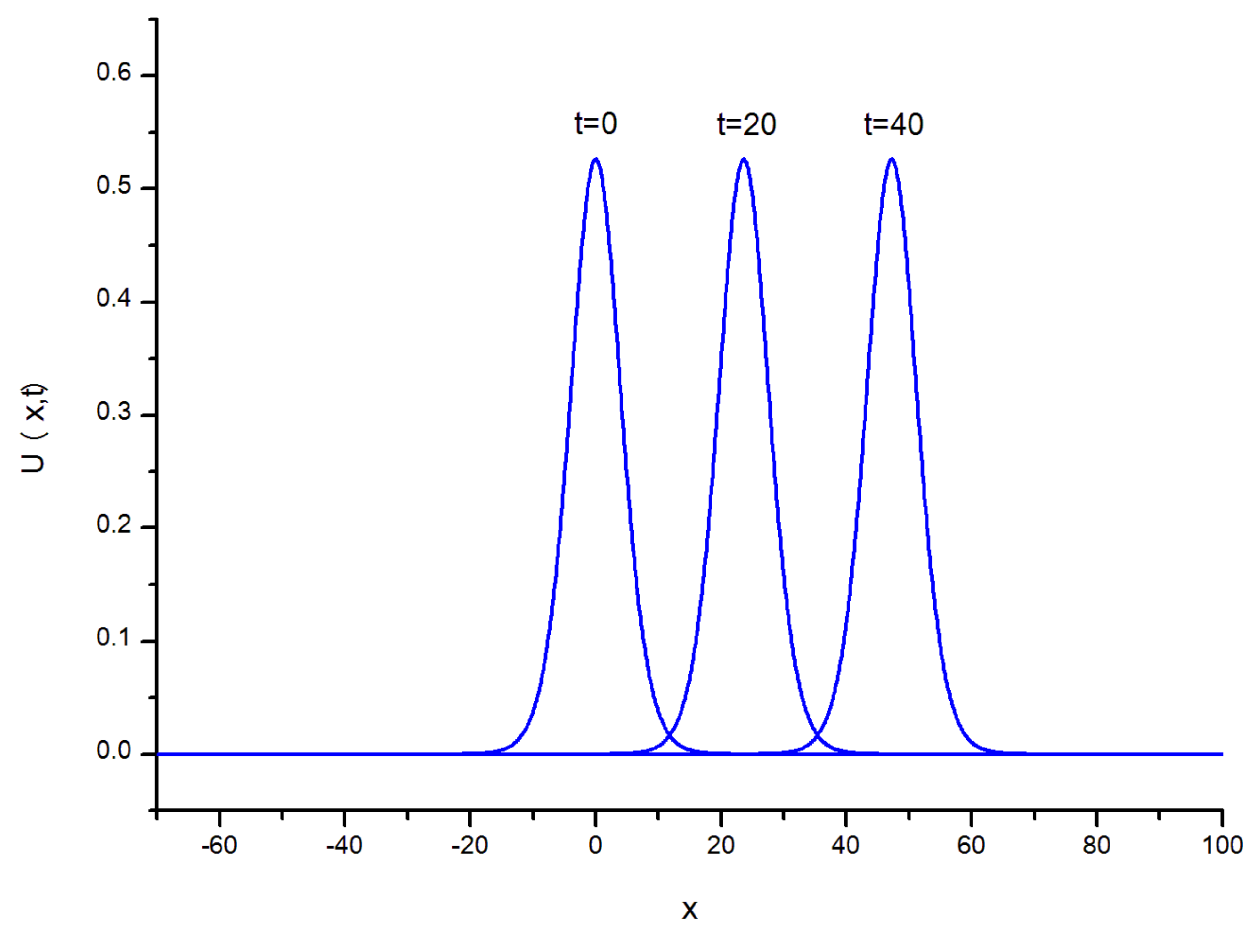

Fig. 1: Single solitary wave with $h=0.1, \Delta t=0.1,-70 \leq x \leq 100, t=0,20$ and 40 .

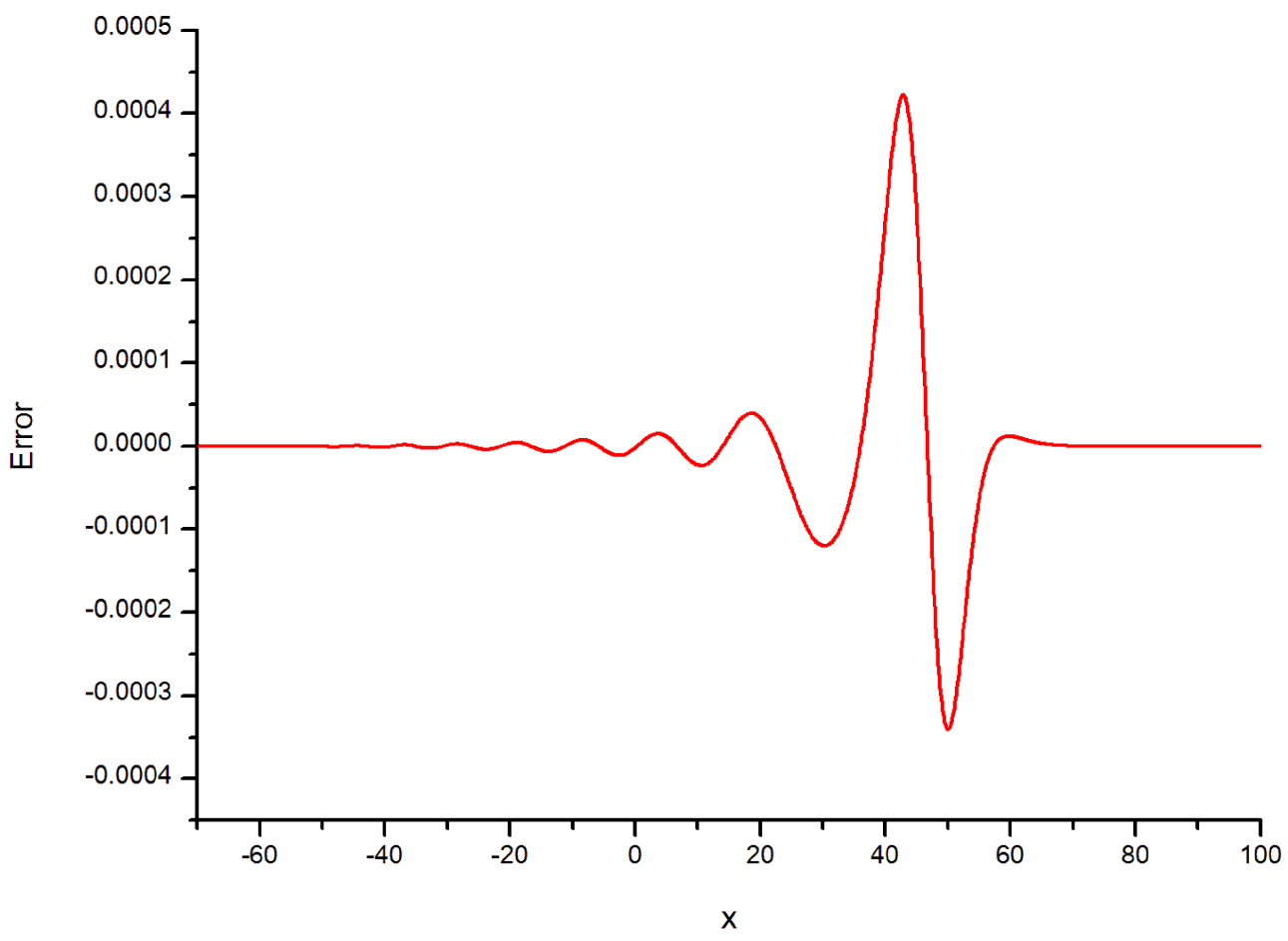

Fig. 2: Error $h=0.1, \Delta t=0.1,-70 \leq x \leq 100, t=40$. 


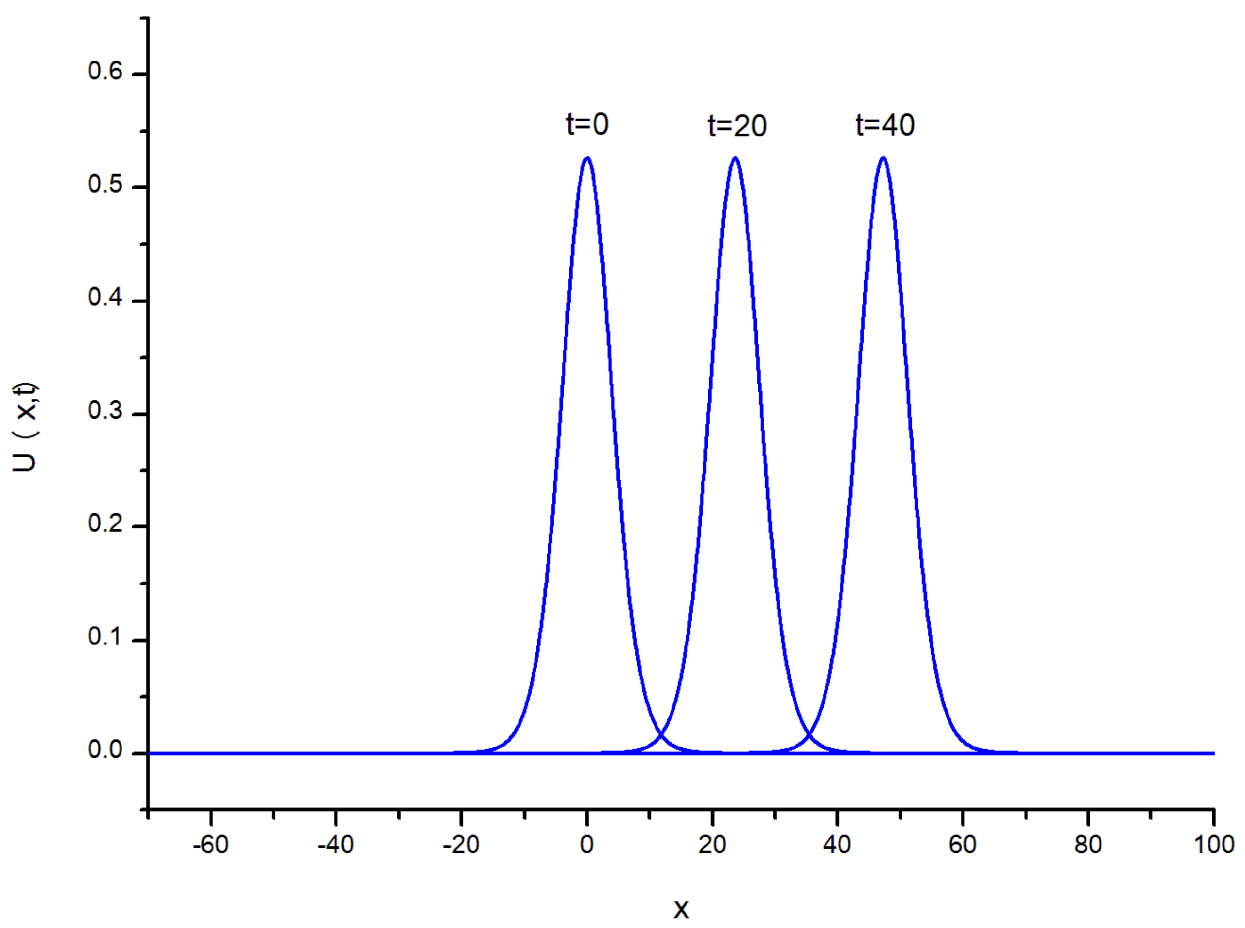

Fig. 3: Single solitary wave with $h=0.05, \Delta t=0.05,-70 \leq x \leq 100, t=0,20$ and 40 .

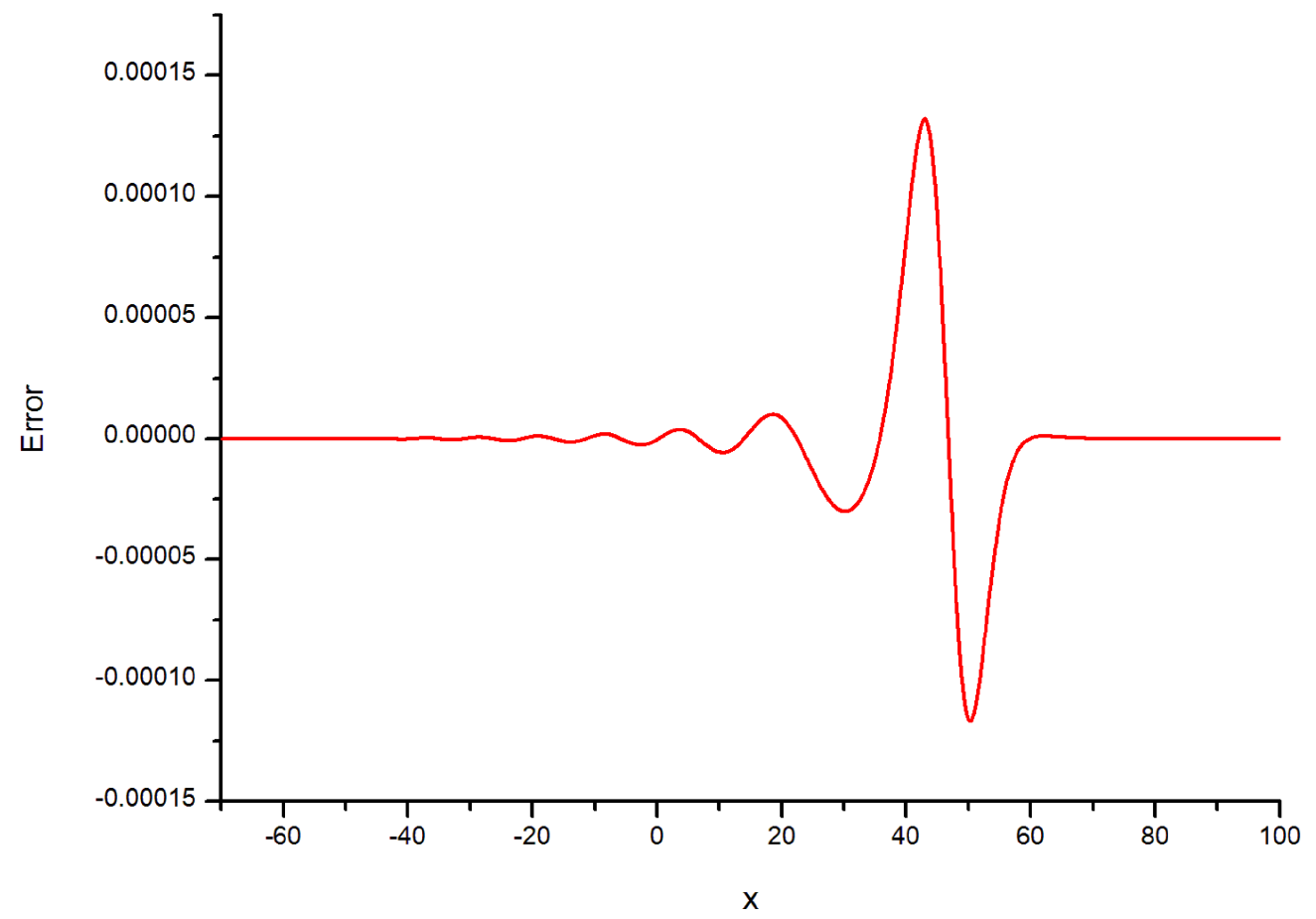

Fig. 4: Error $h=0.05, \Delta t=0.05,-70 \leq x \leq 100, t=40$. 
Table 2: Comparison of the results for single solitary wave with amplitude $=0.52632, h=0.05, \Delta t=0.05,-70 \leq x \leq$ 100.

\begin{tabular}{ccccccc}
\hline & $t$ & 0 & 10 & 20 & 30 & 40 \\
\hline$I_{1}$ & Present & 5.4981692134 & 5.4981691962 & 5.4981691829 & 5.4981691736 & 5.4981691629 \\
& {$[29]$} & 5.4980606845 & 5.4980608372 & 5.4980610805 & 5.4980612870 & 5.4980613985 \\
\hline$I_{2}$ & Present & 1.9897831853 & 1.9897831854 & 1.9897831852 & 1.9897831856 & 1.9897831853 \\
& {$[29]$} & 1.9843901753 & 1.9844010295 & 1.9844143675 & 1.9844232703 & 1.9844289740 \\
\hline$L_{2} \times 10^{4}$ & Present & 0.000000 & 0.854386 & 1.779040 & 2.810186 & 3.783328 \\
& {$[29]$} & 0.000000 & 4.113510 & 7.631169 & 10.62971 & 13.27645 \\
& {$[30]$} & - & - & 1.64418 & - & - \\
& {$[30]$} & - & - & 1.10984 & - & - \\
\hline$L_{\infty} \times 10^{4}$ & Present & 0.000000 & 0.343706 & 0.627075 & 0.975412 & 1.293116 \\
& {$[29]$} & 0.000000 & 1.582641 & 2.835874 & 3.843906 & 4.709118 \\
& {$[30]$} & - & - & 0.635011 & - & - \\
& {$[30]$} & - & - & 0.309342 & - & - \\
\hline
\end{tabular}

Table 3: Comparison of the results for single solitary wave with amplitude $=0.52632, h=0.025, \Delta t=0.025,-70 \leq x \leq$ 100.

\begin{tabular}{|c|c|c|c|c|c|c|}
\hline \multicolumn{2}{|c|}{$\bar{t}$} & 0 & 10 & 20 & 30 & 40 \\
\hline \multirow[t]{2}{*}{$I_{1}$} & Present & 5.4981698357 & 5.4981697751 & 5.4981697199 & 5.4981696708 & 5.4981696247 \\
\hline & [29] & 5.4981454184 & 5.4981454791 & 5.4981455454 & 5.498146095 & 5.4981456591 \\
\hline \multirow[t]{2}{*}{$I_{2}$} & Present & 1.9897809061 & 1.9897809063 & 1.9897809028 & 1.9897808998 & 1.9897808987 \\
\hline & [29] & 1.9849493353 & 1.9843521098 & 1.9843555206 & 1.9843578113 & 1.9843592922 \\
\hline \multirow[t]{4}{*}{$L_{2} \times 10^{4}$} & Present & 0.000000 & 0.351702 & 0.916735 & 1.043479 & 1.183139 \\
\hline & [29] & 0.000000 & 1.028173 & 1.905450 & 2.650990 & 3.306738 \\
\hline & [30] & - & - & 0.411082 & - & - \\
\hline & [30] & - & - & 0.277477 & - & - \\
\hline \multirow[t]{4}{*}{$L_{\infty} \times 10^{5}$} & Present & 0.000000 & 1.420544 & 3.258903 & 4.681364 & 4.847163 \\
\hline & [29] & 0.000000 & 3.965867 & 7.097948 & 9.610332 & 11.76011 \\
\hline & [30] & - & - & 1.58769 & - & - \\
\hline & [30] & - & - & 0.773365 & - & - \\
\hline
\end{tabular}

Finally, for the third set, the parameters $h=0.025$ and $\Delta t=0.025$ with range $[-70,100]$ are chosen to compare the results obtained by the present method with earlier results [29,30]. So, the solitary wave has amplitude 0.52632 and the computations are done until time $t=40$ to obtain the invariants and the error norms $L_{2}$ and $L_{\infty}$ at various times. Error norms $L_{2}$ and $L_{\infty}$ and conserved quantities are tabulated in Table 3 together with earlier results [29]. It is clearly seen from the table the error norms obtained by the present method are smaller than given in Ref.[29] and almost te same in Ref.[30] at $t=20$. The agreement between numerical and analytic solutions is perfect. Percentage of relative changes of $I_{1}$ and $I_{2}$ are found to be $3.8382 \times 10^{-6} \%, 3.7 \times 10^{-7} \%$, respectively. It is clear that the soliton moves to the right at a constant speed and almost unchanged amplitude as time increases, as expected. The profiles of the solitary wave at different time levels are depicted in Figure 5. The distributions of the errors at time $t=40$ are shown graphically for solitary waves amplitudes 0.52632 in Figure 6.

\section{Conclusion}

A numerical solution of the Rosenau-KdV equation based on the sextic B-spline subdomain method has been successfully presented. The performance of the method is examined well by studying the propagation of a single solitary wave. The 




Fig. 5: Single solitary wave with $h=0.025, \Delta t=0.025,-70 \leq x \leq 100, t=0,20$ and 40 .

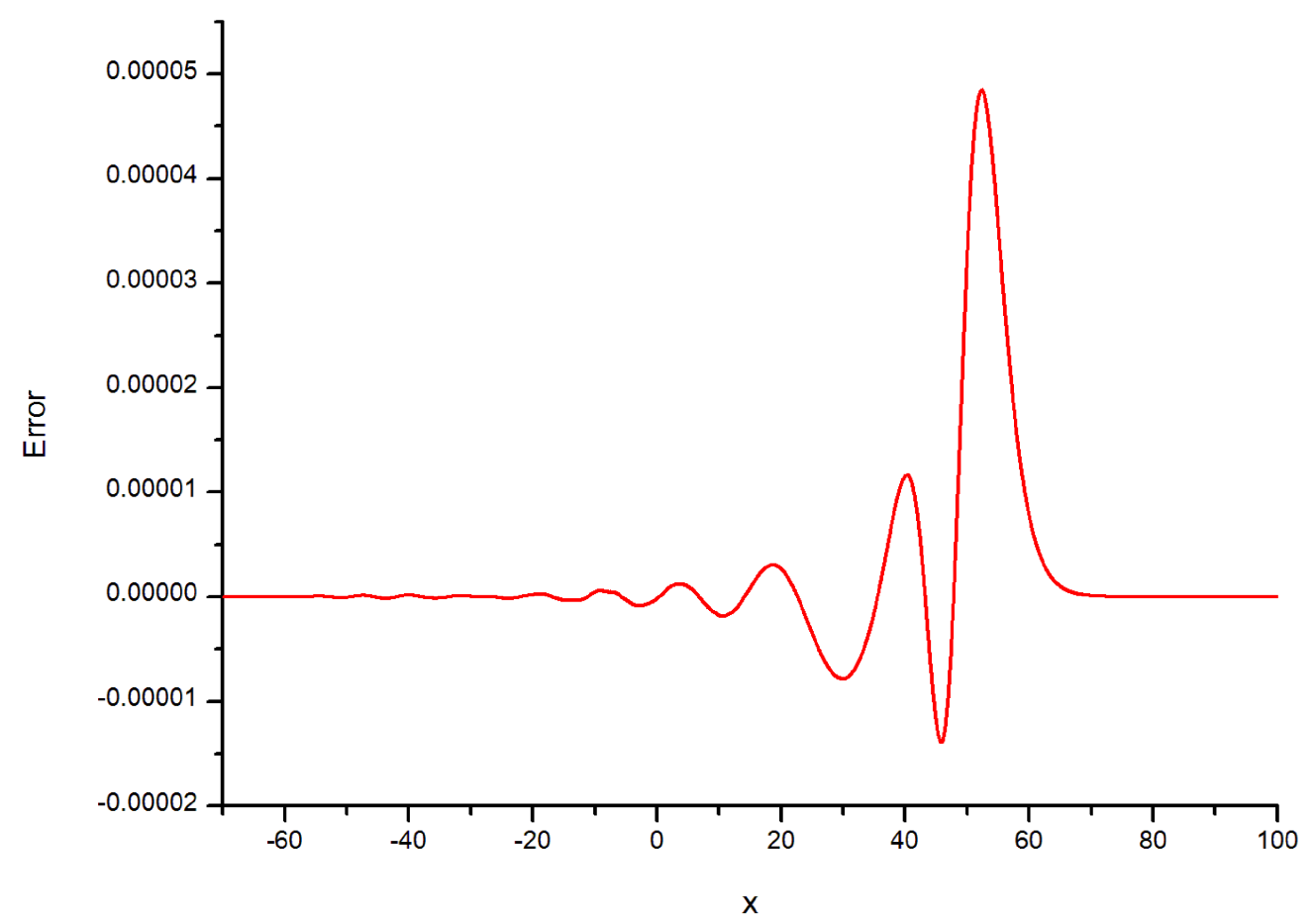

Fig. 6: Error $h=0.025, \Delta t=0.025,-70 \leq x \leq 100, t=40$. 
method successfully models for the motion of solitary waves. The nonlinear term of the equation is linearized. A linear stability analysis based on the von-Neumann theory indicated that the numerical method is unconditionally stable. Three test examples are studied to examine the performance of the scheme. To show how good and accurate the numerical solutions of the test examples, the error norms $L_{2}$ and $L_{\infty}$ and the invariant quantities $I_{1}$ and $I_{2}$ have been used. It is seen that the error norms are sufficiently small and the invariants are well conserved. The obtained small errors for the solitary wave solution and conservation constants have been kept satisfactorily constant during the computer run. The obtained results show that the present method is more accurate than earlier results in the literature. The result shows that subdomain method is powerful mathematical tool for solving nonlinear partial differential equations having wide applications in physical problem represented by Rosenau-KdV equation.

\section{Acknowledgements}

The author, Turgut Ak, is grateful to The Scientific and Technological Research Council of Turkey for granting scholarship for Ph.D. studies.

\section{References}

[1] M. Antonova and A. Biswas, Adiabatic parameter dynamics of perturbed solitary waves, Communications in Nonlinear Science and Numerical Simulation, 14 (3), 734-748, (2009).

[2] A. Biswas, D. Milovic and A. Ranasinghe, Solitary waves of Boussinesq equation in a power law media, Communications in Nonlinear Science and Numerical Simulation, 14 (11), 3738-3742, (2009).

[3] A. Biswas and M. S. Ismail, 1-Soliton solution of the coupled KdV equation and Gear-Grimshaw model, Applied Mathematics and Computation, 216 (12), 3662-3670, (2010).

[4] A. H. Bhrawy, M. A. Abdelkawy and A. Biswas, Cnoidal and snoidal wave solutions to coupled nonlinear wave equations by the extended Jacobi's elliptic function method, Communications in Nonlinear Science and Numerical Simulation, 18 (4), 915-925, (2013).

[5] A. H. Bhrawy, M. A. Abdelkawy, S. Kumar and A. Biswas, Solitons and other solutions to Kadomtsev-Petviashvili equation of B-type, Romanian Journal of Physics, 58 (7-8), 729-748, (2013).

[6] G. Ebadi, N. Y. Fard, A. H. Bhrawy, S. Kumar, H. Triki, A. Yildirim and A. Biswas, Solitons and other solutions to the (3+1)dimensional extended Kadomtsev-Petviashvili equation with power law nonlinearity, Romanian Reports in Physics, 65 (1), 27-62, (2013).

[7] H. Triki, A. H. Kara, A. Bhrawy and A. Biswas, Soliton solution and conservation law of Gear-Grimshaw model for shallow water waves, Acta Physica Polonica A, 125 (5), 1099-1106, (2014).

[8] H. Triki, Z. Jovanoski and A. Biswas, Dynamics of two-layered shallow water waves with coupled KdV equations, Romanian Reports in Physics, 66 (2), 274-285, (2014).

[9] H. Triki, M. Mirzazadeh, A. H. Bhrawy, P. Razborova and A. Biswas, Soliton and other solutions to long-wave short-wave interaction equation, Romanian Journal of Physics, 60 (1-2), 72-86, (2015).

[10] M. Mirzazadeh, M. Eslami and A. Biswas, 1-Soliton solution to KdV6 equation, Nonlinear Dynamics, 80 (1-2), 387-396, (2015).

[11] D. J. Korteweg, G. de Vries, On the change of form of long waves advancing in a rectangular canal, and on a new type of long stationary wave, Philosophical Magazine, 39, 442-443, (1895).

[12] Y. Cui and D.-K. Mao, Numerical method satisfying the first two conservation laws for the Korteweg-de Vries equation, Journal of Computational Physics, 227 (1), 376-399, (2007).

[13] S. Zhu and J. Zhao, The alternating segment explicit-implicit scheme for the dispersive equation, Applied Mathematics Letters, 14 (6), 657-662, (2001). 
[14] M. M. Rashidi, D. D. Ganji, S. Dinarvand, Explicit analytical solutions of the generalized Burger and Burger-Fisher equations by homotopy perturbation method, Numerical Methods for Partial Differential Equations, 25 (2), 409-417, (2009).

[15] M. M. Rashidi, E. Erfani, New Analytical Method for Solving Burgers' and Nonlinear Heat Transfer Equations and Comparison with HAM, Computer Physics Communications, 180 (9), 1539-1544, (2009).

[16] A. Alexandrescu and J. R. Salgueiro, Efficient numerical method for linear stability of solitary waves, Computer Physics Communications, 182 (12), 2479-2485, (2011).

[17] P. Rosenau, A quasi-continuous description of a nonlinear transmission line, Physica Scripta, 34, 82-829, (1986).

[18] P. Rosenau, Dynamics of dense discrete systems, Progress of Theoretical Physics, 79, 1028-1042, (1988).

[19] S. K. Chung and A. K. Pani, Numerical methods for the Rosenau equation, Applicable Analysis, 77 (3-4), 351-369, (2011).

[20] K. Omrani, F. Abidi, T. Achouri, and N. Khiari, A new conservative finite difference scheme for the Rosenau equation, Applied Mathematics and Computation, 201 (1-2), 35-43, (2008).

[21] J. Hu and K. Zheng, Two conservative difference schemes for the generalized Rosenau equation, Boundary Value Problems, (2010), Article ID 543503, 18 pages.

[22] S. A. V. Manickam, A. K. Pani, and S. K. Chung, A second-order splitting combined with orthogonal cubic spline collocation method for the Rosenau equation, Numerical Methods for Partial Differential Equations, 14 (6), 695-716, (1998).

[23] Y. D. Kim and H. Y. Lee, The convergence of finite element Galerkin solution for the Roseneau equation, The Korean Journal of Computational and Applied Mathematics, 6 (1), 1-13, (1999).

[24] J.-M. Zuo, Solitons and periodic solutions for the Rosenau-KdV and Rosenau-Kawahara equations, Applied Mathematics and Computation, 215 (2), 835-840, (2009).

[25] A. Esfahani, Solitary wave solutions for generalized Rosenau-KdV equation, Communications in Theoretical Physics, 55 (3), 396-398, (2011).

[26] P. Razborova, H. Triki, and A. Biswas, Perturbation of dispersive shallow water waves, Ocean Engineering, 63, 1-7, (2013).

[27] G. Ebadi, A. Mojaver, H. Triki, A. Yildirim, and A. Biswas, Topological solitons and other solutions of the Rosenau-KdV equation with power law nonlinearity, Romanian Journal of Physics, 58 (1-2), 1-10, (2013).

[28] A. Saha, Topological 1-soliton solutions for the generalized Rosenau-KdV equation, Fundamental Journal of Mathematical Physics, 2 (1), 19-23, (2012).

[29] J. Hu, Y. Xu, and B. Hu, Conservative linear difference scheme for Rosenau-KdV equation, Advances in Mathematical Physics, (2013), Article ID 423718, 7 pages.

[30] B. Wongsaijai and K. Poochinapan, A three-level average implicit finite difference scheme to solve equation obtained by coupling the Rosenau-KdV equation and the Rosenau-RLW equation, Applied Mathematics and Computation, 245, 289-304, (2014).

[31] P. M. Prenter, Splines and Variational Methods, J. Wiley, New York, (1975). 